\title{
Dynamically generated open-charm baryons beyond the zero-range approximation
}

\author{
C. E. Jiménez-Tejero, ${ }^{1}$ A. Ramos, ${ }^{1}$ and I. Vidaña ${ }^{2}$ \\ ${ }^{1}$ Departament d'Estructura i Constituents de la Matèria and Institut de Ciències del Cosmos, Universitat de Barcelona, \\ Avda. Diagonal 647, E-08028 Barcelona, Spain \\ ${ }^{2}$ Centro de Física Computacional, Department of Physics, University of Coimbra, 3004-516 Coimbra, Portugal
}

(Received 30 July 2009; revised manuscript received 16 October 2009; published 18 November 2009)

\begin{abstract}
The interaction of the low-lying pseudoscalar mesons with the ground-state baryons in the charm sector is studied within a coupled-channel approach using a $t$-channel vector-exchange driving force. The amplitudes describing the scattering of the pseudoscalar mesons off the ground-state baryons are obtained by solving the Lippmann-Schwinger equation. We analyze in detail the effects of going beyond the $t=0$ approximation. Our model predicts the dynamical generation of several open-charm baryon resonances in different isospin and strangeness channels, some of which can be clearly identified with recently observed states.
\end{abstract}

DOI: 10.1103/PhysRevC.80.055206

PACS number(s): 14.20.Lq, 14.40.Lb, 12.38.Lg, 24.85.+p

\section{INTRODUCTION}

A very active topic of research in hadron physics is the study and characterization of resonances to establish whether they qualify as genuine $q \bar{q}$ or $q q q$ states or, alternatively, behave more as hadron molecules generated dynamically. A series of pioneer works [1-6], based on a $t$-channel vector-meson exchange force, already predicted a wealth of $s$-wave baryon resonances generated by coupled-channel dynamics with effective hadronic degrees of freedom rather than quarks and gluons. The earlier approaches have been adapted in the past decade to the modern language of chiral Lagrangians [7-24], and many resonances in the light SU(3) sector, which cannot be described properly by quark models [25] unless substantial meson-baryon components are included [26], have been identified with dynamical states generated from the interactions of mesons of the pseudoscalar $0^{-}$octet with the $1 / 2^{+}$ground-state baryons. Some consequences of these studies, such as the two-pole nature of $\Lambda(1405)$, have been confirmed through analyses [27,28] of different experimental reactions [29-31]. Note that the basic structure of a molecular-type baryon is quite different than that implied by the quark models, even when the models include the dressing with meson-baryon components, as in the ${ }^{3} P_{0}$ formalism, in which the $q q q$ Hilbert space is coupled to the meson-baryon Hilbert space through the creation of a $q \bar{q}$ pair [32-37]. In the former case, the degrees of freedom are purely hadronic and the resonances must be seen as pseudobound states of two hadrons, whereas in the latter case, the essential component of a baryon is still of three-quark nature. A goal in hadron physics research is to distinguish between both pictures by thorough analyses of as many properties of the hadron as possible, such as the mass, width, magnetic momenta, and form factors.

In recent years, it has been demonstrated that besides the $s$-wave baryon resonances many more states can be generated dynamically. Baryon resonances with $J^{P}=3 / 2^{-}$ were studied based on the leading-order chiral Lagrangian with the decuplet $3 / 2^{+}$fields [38-41]. $D$-wave baryon resonances were also generated dynamically with vector-meson degrees of freedom in Refs. [42-47]. Another promising line of research is the recent interpretation of low-lying $J^{P}=1 / 2^{+}$ resonances as molecular states of two pseudoscalar mesons and one baryon [48-52]. All these results support the socalled hadrogenesis conjecture, formulated a few years ago by Lutz and Kolomeitsev, according to which resonances not belonging to the large- $N_{c}$ ground state of QCD are generated by coupled-channel dynamics [18,42,53-55].

The study of charmed hadrons is receiving increased attention thanks to the efforts of a series of collaborations at lepton colliders (CLEO, BELLE, BaBar) and hadron facilities (CDF at Fermilab, PHENIX and STAR at RHIC, FAIR at GSI). The new results not only confirm, with better statistics, previously seen charmed states but also give rise to the discovery of many new hadrons [56-69]. Coupled-channel unitary schemes have been recently extended to include the charm degree of freedom and have been applied to the description of open- and hidden-charm mesons, with the observation that some states admit a straightforward interpretation as meson molecules [70-75]. Similar methods have been used for describing baryons with charm (denoted here as $C$ ), motivated in part by a clear parallelism between the behavior of $\Lambda(1405)$ in the $C=0, S=-1$ sector with the behavior of $\Lambda_{c}(2595)$ in the $C=1, S=0$ sector [76-81]. To be consistent with the spin-flavor heavy-quark symmetry that develops in this heavy sector [82-84], the vector mesons and $J=3 / 2^{+}$baryons have recently been included in the basis of meson-baryon states, employing a static spin-flavor SU(8) scheme [85] similar to that developed in the light sector $[43,44]$. Treating the $D$ and $D^{*}$ mesons equally has led to the observation that some of the dynamically generated states, such as $\Lambda_{c}(2595)$, have mostly a $D^{*} N$ composition rather than $D N$ molecular nature. In any case, the fact that some of the dynamically generated charmed hadrons can be readily identified with observed resonances, such as the $J^{P}=1 / 2^{-} \Lambda_{c}(2595)$ or the $J^{P}=3 / 2^{-} \Lambda_{c}(2625)$ charmed baryons, sustains the hadrogenesis conjecture [18,42,53-55].

Apart from the different bases of states included in the models, a common feature of all the previous works is the use of an interaction based on the $t$-channel exchange of vector mesons, as the driving force for the $s$-wave scattering of pseudoscalar mesons off ground-state baryons. The limit $t \rightarrow 0$ 
is applied, leading to a vector-type Weinberg-Tomozawa (WT) zero-range interaction. This procedure is justified for on-shell meson-baryon transitions, $M B \rightarrow M^{\prime} B^{\prime}$, which are diagonal $\left(M^{\prime} B^{\prime}=M B\right)$, and hence the value of $t$ is small as long as one is not too far from the threshold. It also holds for nondiagonal amplitudes $\left(M^{\prime} B^{\prime} \neq M B\right)$ that show a moderate difference of masses between the initial and final mesons and baryons involved, as is the case of meson-baryon scattering within the light SU(3) world. However, the coupled-channel dynamics in the heavy sector also has charm-exchange processes for which the difference of masses between the external mesons is comparable with the mass of the charmed vector meson being exchanged. This clearly signals the breakdown of the zero-range approximation, which is no longer reliable for these nondiagonal transitions. Although one may still argue that many of the dynamically generated states are triggered by a single dominant meson-baryon interaction component, and hence their energy can be well estimated by the pole position of an uncoupled calculation involving only diagonal amplitudes, the corresponding width will be determined by nondiagonal amplitudes and will therefore depend on whether the $t=0$ approximation is implemented. Moreover, it is well known that some resonances owe their origin to a particularly strong coupling between different channels, hence involving nondiagonal transitions, in which case the $t=0$ approximation is not at all appropriate for these states.

In the present work, we study the charmed baryon resonances obtained dynamically from the interaction of the low-lying pseudoscalar mesons with the ground-state baryons within a coupled-channel approach, using the full $t$-dependence of the $t$-channel vector-exchange driving term, instead of the $t=0$ approximation. We incorporate the $t$ dependence within a general four-dimensional integration scheme, which we reduce to a three-dimensional equation of the Lippmann-Schwinger type, and analyze in detail the effects of going beyond the $t=0$ approximation within this scheme.

The article is organized as follows. In Sec. II, we present the formalism, showing the details of the kernel employed and the equation used to obtain the scattering amplitudes. Our results for the properties of the baryon resonances with charm in various strangeness and isospin cases are shown in Sec. III, where we also compare the $t=0$ results. A summary of our conclusions is presented in Sec. IV.

\section{FORMALISM}

Following the original work of Hofmann and Lutz [78], we identify a $t$-channel exchange of vector mesons as the driving force for the $s$-wave scattering. In their original model, Hofmann and Lutz exploited the universal vector-meson coupling hypothesis. They considered the $t$-channel exchange of vector mesons between pseudoscalar mesons in 16-plet and baryons in 20-plet representations in such a way as to respect chiral symmetry for the light-meson sector. The Weinberg-Tomozawa interaction is recovered in the zero-range limit (i.e., $t \rightarrow 0$; see Eqs. (6) and (8) of Ref. [78] for details).
The scattering kernel has the form

$$
\begin{aligned}
& V_{i j}^{(I, S, C)}\left(k_{i}, q_{i}, k_{j}, q_{j}\right) \\
& =\frac{g^{2}}{4} \sum_{V \in[16]} C_{i j ; V}^{(I, S, C)} \bar{u}\left(p_{j}\right) \gamma^{\mu}\left(g_{\mu \nu}-\frac{\left(q_{i}-q_{j}\right)_{\mu}\left(q_{i}-q_{j}\right)_{v}}{m_{V}^{2}}\right) \\
& \quad \times \frac{1}{t-m_{V}^{2}}\left(q_{i}+q_{j}\right)^{v} u\left(p_{i}\right),
\end{aligned}
$$

where the sum runs over all vector mesons of the SU(4) 16-plet; $\left(\rho, K^{*}, \bar{K}^{*}, \omega, \phi, D^{*}, D_{s}^{*}, \bar{D}^{*}, \bar{D}_{s}^{*}, J / \Psi\right) ; m_{V}$ is the mass of the exchanged vector meson; $g$ is the universal vector-meson coupling constant; $k_{i}, q_{i}, k_{j}$, and $q_{j}$ are the four momenta of the incoming and outgoing baryon and meson; and the coefficients $C_{i j ; V}^{(I, S, C)}$ denote the strength of the interaction in the different sectors (isospin, strangeness, charm) $(I, S, C=1)$ and channels $(i, j)$. The value of $g=6.6$ reproduces the decay width of the $\rho$ meson [86].

Assuming that $\left|t / m_{V}^{2}\right| \ll 1$, we can neglect the $t$ dependence of the scattering kernel, giving rise to the zero-range approximation. The $s$-wave projection of the scattering kernel under such approximation is easily obtained, and in the center-of-mass (c.m.) frame it takes the form

$$
\begin{aligned}
& V_{i j, l=0}^{(I, S, C)}\left(k_{i}, q_{i}, k_{j}, q_{j}\right) \\
& =-N \frac{g^{2}}{4} \sum_{V \in[16]} \frac{C_{i j ; V}^{(I, S, C)}}{m_{V}^{2}}\left[\omega\left(\left|\vec{k}_{i}\right|\right)+E\left(\left|\vec{k}_{i}\right|\right)+\omega\left(\left|\vec{k}_{j}\right|\right)\right. \\
& \left.\quad+E\left(\left|\vec{k}_{j}\right|\right)-M_{i}-M_{j}-\frac{m_{j}^{2}-m_{i}^{2}}{m_{V}^{2}}\left(M_{i}-M_{j}\right)\right],
\end{aligned}
$$

where $m_{i}, m_{j}, M_{i}, M_{j}$ are the masses of the incoming and outgoing mesons and baryons and $\omega_{i}\left(\left|\vec{k}_{i}\right|\right), \omega_{j}\left(\left|\vec{k}_{j}\right|\right), E_{i}\left(\left|\vec{k}_{i}\right|\right)$, $E_{j}\left(\left|\vec{k}_{j}\right|\right)$ are their corresponding energies, which have been taken to be their on-shell values. The factor $N=\left\{\left[E\left(\left|\vec{k}_{i}\right|\right)+\right.\right.$ $\left.\left.M_{i}\right]\left[E\left(\left|\vec{k}_{j}\right|\right)+M_{j}\right] /\left(4 M_{i} M_{j}\right)\right\}^{1 / 2}$ comes from the normalization of the Dirac spinors. Note that for physical (fully on-shell) transition amplitudes, one has $\omega_{i}\left(\left|\vec{k}_{i}\right|\right)+E_{i}\left(\left|\vec{k}_{i}\right|\right)=\omega_{j}\left(\left|\vec{k}_{j}\right|\right)+$ $E_{j}\left(\left|\vec{k}_{j}\right|\right)=\sqrt{s}$, in which case one recovers the familiar expression of the commonly used Weinberg-Tomozawa interaction. The last term in Eq. (2) is usually ignored in most works, to be more consistent with the $t=0$ approximation applied in the denominator of the meson-exchange propagator, as noted in Ref. [81]. In any case, its consideration introduces only minor corrections [78].

To illustrate the validity of this approach, we show in Fig. 1 the value of $t / m_{V}^{2}$ for $\cos \theta=-1$ as a function of $\sqrt{s}$, where $m_{V}$ is the mass of a representative meson exchanged, which we take to be the $\rho$ meson mass for diagonal transitions and the $D^{*}$ meson mass for charm-exchange ones. The range of energies goes roughly between the $\pi \Sigma_{c}$ and $D N$ thresholds, thereby covering the region of the $J^{P}=1 / 2^{-}$resonance $\Lambda_{c}(2595)$, which is a prime example of a dynamically generated opencharm baryon state in various approaches [76-81]. It also expands beyond the $D N$ threshold for about $300 \mathrm{MeV}$ to explore the energy region that will be relevant in future studies of the $D$-meson self-energy in a nuclear medium. As one 


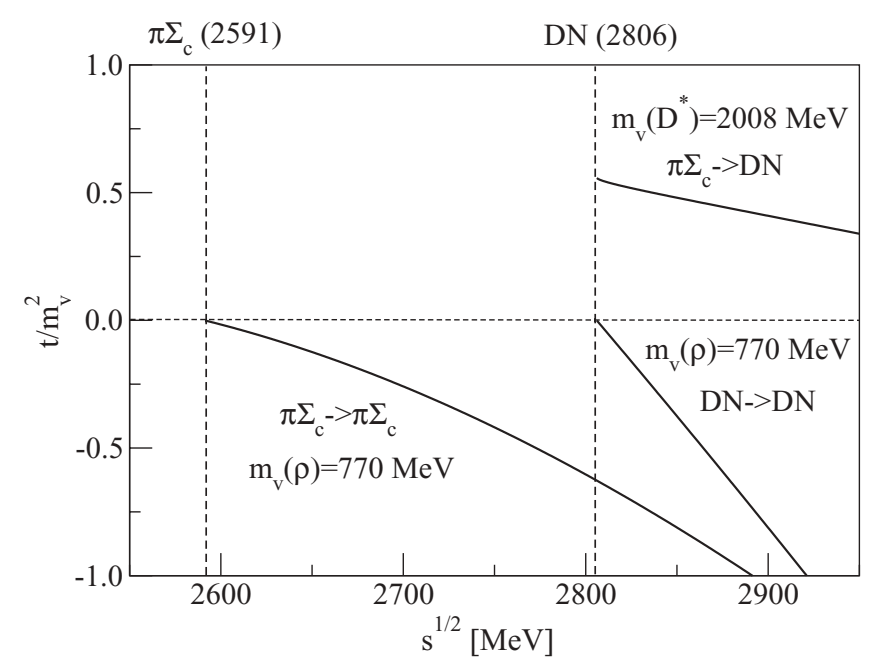

FIG. 1. Dependence on the c.m. energy $\sqrt{s}$ of the fourmomentum transfer $t / m_{V}^{2}$ for $\cos \theta=-1$ and for different transition amplitudes.

can see, the value of $t / m_{V}^{2}$ is only close to zero for diagonal transitions around their corresponding energy threshold, and its size can be comparable to one at the energies of interest. For the nondiagonal $\pi \Sigma_{c} \rightarrow D N$ transition, $t / m_{V}^{2}$ never goes to zero and acquires values of the order of 0.5 .

The results of Fig. 1 point clearly to the need to explore the effects of going beyond the $t=0$ approximation, an attempt that is taken in the present work by considering the full $t$-dependence of the scattering kernel given by Eq. (1). By performing the $s$-wave projection, we obtain the following analytic expression:

$$
\begin{aligned}
& V_{i j, l=0}^{(I, S, C)}\left(\vec{k}_{i}, \vec{k}_{j}\right) \\
& =N \frac{g^{2}}{8} \sum_{V \in[16]} C_{i j ; V}^{(I, S, C)}\left[\frac{2 \beta}{b}+\frac{\alpha b-\beta a}{b^{2}} \ln \left(\frac{a+b}{a-b}\right)\right],
\end{aligned}
$$

with $a, b, \alpha$, and $\beta$ being

$$
\begin{aligned}
a= & m_{i}^{2}+m_{j}^{2}-2 \omega_{i}\left(\left|\vec{k}_{i}\right|\right) \omega_{j}\left(\left|\vec{k}_{j}\right|\right)-m_{V}^{2}, \\
b= & 2\left|\vec{k}_{i}\right|\left|\vec{k}_{j}\right|, \\
\alpha= & \Omega_{i}\left(\left|\vec{k}_{i}\right|\right)+\Omega_{j}\left(\left|\vec{k}_{j}\right|\right)-M_{i}-M_{j}-\frac{m_{j}^{2}-m_{i}^{2}}{m_{V}^{2}}\left[\Omega_{j}\left(\left|\vec{k}_{j}\right|\right)\right. \\
& \left.-\Omega_{i}\left(\left|\vec{k}_{i}\right|\right)+M_{i}-M_{j}\right], \\
\beta= & \frac{\left|\vec{k}_{i}\right|\left|\vec{k}_{j}\right|}{\left[E_{i}\left(\left|\vec{k}_{i}\right|\right)+M_{i}\right]\left[E_{j}\left(\left|\vec{k}_{j}\right|\right)+M_{j}\right]}\left(\Omega_{i}\left(\left|\vec{k}_{i}\right|\right)+\Omega_{j}\left(\left|\vec{k}_{j}\right|\right)\right. \\
& +M_{i}+M_{j}-\frac{m_{j}^{2}-m_{i}^{2}}{m_{V}^{2}}\left[\Omega_{j}\left(\left|\vec{k}_{j}\right|\right)-\Omega_{i}\left(\left|\vec{k}_{i}\right|\right)\right. \\
& \left.\left.+M_{j}-M_{i}\right]\right),
\end{aligned}
$$

where we have defined $\Omega(|\vec{k}|) \equiv \omega(|\vec{k}|)+E(|\vec{k}|)$.

The $t=0$ expression of the $s$-wave interaction is recovered by expanding the logarithm of Eq. (3) in the limit $b / a \rightarrow 0$ up to the linear term in $b / a$ and setting $a=-m_{V}^{2}$. As one can infer from the values of $t / m_{V}^{2}$ displayed in Fig. 1, keeping the $t$ dependence in the denominator of the kernel [see Eq. (1)] will decrease the strength of the diagonal transitions, because in this case $t<0$. Therefore, to reproduce a given resonance found in local models, in general the present approach will need to compensate for the lack of strength with a greater value of the cutoff momentum used to regularize the loop integrals. However, nondiagonal amplitudes, responsible mainly for the decay width of the dynamically generated states, will be enhanced because they are characterized by a positive time-like $t$ value resulting from the large mass difference between the mesons and baryons involved in the transition. As a consequence, our resonances will be wider than those found in the local models.

Retaining the $t$ dependence in the kernel implies additional analytical structures [87] that prevent us from obtaining the scattering amplitudes by solving the Bethe-Salpeter (BS) equation using only on-shell amplitudes. Instead, we incorporate the $t$ dependence within a more general four-dimensional integration scheme, which we reduce to a three-dimensional equation of the Lippmann-Schwinger type. To this end, for a given value of the total scattering energy, we evaluate the transition potential between any arbitrary pair of relative momenta within the cutoff value, keeping the dependence on the momentum transfer in the corresponding matrix element. This procedure follows the same spirit of the usual mesonexchange models of the $N N$ interaction [88], also applied to meson-baryon scattering models $[89,90]$. We note that keeping the full $t$ dependence in the exchanged meson propagator also implies that retardation effects are implemented, as done in the three-dimensional reduction of the BS equation of Ref. [91], which is different than the prescription based on time-dependent perturbation theory [88]. Both choices differ in the way off-shell effects are implemented, and their differences in the $N N$ sector show up especially in the contributions of the two-meson-exchange box diagrams [88], not included in the present model. In any case, observables can be matched to experimental data with either choice of retardation effects by selecting appropriate values of the renormalization parameters.

In Eqs. (1)-(3), we assume infinitely lived (zero-width) exchanged vector mesons although some of them have large widths as a result of their strong decay into a pair of mesons, such as the $\rho$ meson contributing to diagonal channels. However, we have checked that the value of $t$ is never larger than the square of the minimum energy required for the meson to decay, namely $\left(2 m_{\pi}\right)^{2}$ in the case of $\rho$ exchange or $\left(m_{\pi}+m_{K}\right)^{2}$ for $K^{*}$. In other words, the mesons being exchanged in this problem are largely off shell, and they will be treated as stable particles.

Once the scattering kernel has been constructed, we can obtain the $T$ matrices, which describe the scattering of the pseudoscalar meson fields off the baryon fields, by solving the well-known Lippmann-Schwinger equation:

$$
\begin{aligned}
T_{i j, l=0}^{(I, S, C)}\left(\vec{k}_{i}, \vec{k}_{j}, \sqrt{s}\right) \\
=V_{i j, l=0}^{(I, S, C)}\left(\vec{k}_{i}, \vec{k}_{j}\right)+\sum_{m} \int \frac{d \vec{k}}{(2 \pi)^{3}} F(|\vec{k}|) V_{i m, l=0}^{(I, S, C)}\left(\vec{k}_{i}, \vec{k}\right) \\
\quad \times J_{m}(\sqrt{s}, \vec{k}) T_{m j, l=0}^{(I, S, C)}\left(\vec{k}, \vec{k}_{j}, \sqrt{s}\right),
\end{aligned}
$$


where

$$
\begin{aligned}
& J_{m}^{(I, S, C)}(\sqrt{s}, \vec{k}) \\
& \quad=\frac{M_{m}}{2 E_{m}(|\vec{k}|) \omega_{m}(|\vec{k}|)} \frac{1}{\sqrt{s}-E_{m}(|\vec{k}|)-\omega_{m}(|\vec{k}|)+i \eta} .
\end{aligned}
$$

We have introduced a dipole-type form factor $F(|\vec{k}|)$,

$$
F(|\vec{k}|)=\left(\frac{\Lambda^{2}}{\Lambda^{2}+|\vec{k}|^{2}}\right)^{2},
$$

to regularize the integral. This form is typically adopted in studies of hadron-hadron interactions within the scheme of Lippmann-Schwinger-type equations in the light-flavor sector [88]. The value of the cutoff $\Lambda$ is a free parameter of our model. Given the limited amount of data for charmed-baryon resonances, and to simplify the analysis, the cutoff $\Lambda$ is adjusted to the position of a well-known $J^{P}=1 / 2^{-}$state in a particular isospin and strangeness sector, and the same value is used for the other sectors explored in this work. We also investigate the effect of a Gaussian-type form factor, as well as the dependence of our results on the value of the cutoff employed.

Note that the approaches based on the BS equation solved with on-shell amplitudes ignore the off-shellness (momentum dependence) of the kernel and scattering amplitude in the loop function. Actually, the on-shell factorization can only be justified within the WT form of the potential, which is obtained after applying the $t \rightarrow 0$ limit.

Another aspect worth commenting on is the different extrapolation of the kernel at subthreshold energies. Whereas the kernels of the on-shell BS approaches depend on $\sqrt{s}$ in the form given by Eq. (2), upon replacing $\omega_{i}\left(\left|\vec{k}_{i}\right|\right)+E_{i}\left(\left|\vec{k}_{i}\right|\right)+$ $\omega_{j}\left(\left|\vec{k}_{j}\right|\right)+E_{j}\left(\left|\vec{k}_{j}\right|\right)$ by $2 \sqrt{s}$, the potential used in the threedimensional Lippmann-Schwinger equation depends essentially on the incoming and outgoing three-momenta. These are always taken as real quantities in our approach; hence, the sum of the four single-particle energies is always larger than the sum of the two meson-baryon thresholds involved in the transition. An explicit dependence on $\sqrt{s}$ is only implemented in the meson-baryon intermediate propagator of the LippmannSchwinger equation that determines the scattering amplitudes. Therefore, at subthreshold energies, the factor in the numerator of the kernel used in on-shell BS approaches is smaller than in the present work. This compensates in part for the enhancement in diagonal transitions associated with the $t \rightarrow 0$ limit. In any case, the free parameters of the model (cutoff values) can finally be conveniently fine-tuned to adjust the energy position of a well-known resonance, as is usually done for the case of the $\Lambda_{c}(2595)$, appearing about $200 \mathrm{MeV}$ less than the threshold of the channel $D N$ to which it couples very strongly. In this respect, the differences between the present work and the on-shell BS approaches is more evident in properties tied to nondiagonal transitions, which are reduced in the $t \rightarrow 0$ limit, as is the case of the resonance widths.

To associate a given enhancement of the scattering amplitude to a resonance, we look for a characteristic pole in the unphysical sheet of the complex energy plane. Our prescription of unphysical sheet is such that whenever the real part of the complex energy crosses a meson-baryon threshold cut, the sign of the on-shell momentum is changed for this channel and for the already opened ones, as described in detail in Ref. [4]. Once a pole $z_{R}$ is found, its value determines the Breit-Wigner mass $\left(M=\operatorname{Re} z_{R}\right)$ and width $\left(\Gamma=2 \operatorname{Im} z_{R}\right)$ of the resonance, as seen from real energies, if the pole is not too far from the real axis. The couplings of the resonances to the meson-baryon components of a given sector are obtained from the residues of the scattering amplitude since, close to the pole, it can be parametrized in the form

$$
T_{i j, l=0}^{I, S, C}\left(\vec{k}_{i}, \vec{k}_{j}, z\right)=\frac{g_{i} g_{j}}{z-z_{R}} .
$$

Note that, as it stands, the values of the coupling constants of Eq. (8) depend on the particular momentum values chosen in the evaluation of the $T$-matrix element. Because we are only interested in the size of the couplings relative to the various channels, we evaluate them for the case $\vec{k}_{i}=\vec{k}_{j}=0$.

\section{RESULTS AND DISCUSSION}

One of our main interests here is to study the effects of going beyond the $t=0$ approximation in the kernel, as has been customarily done. We have already anticipated in the previous section that in the case of coupled-channel problems involving light- and heavy-flavor particles, this approximation is not always justified.

All the possible sectors with charm $C=1$ that can be built from the $s$-wave scattering of pseudoscalar mesons with $J^{P}=1 / 2^{+}$baryons are shown in Table I, together with the corresponding meson-baryon coupled channels. In this work, we first study the cases in which some resonance with either $J^{P}=1 / 2^{-}$or unknown spin parity has already been observed. This includes the sectors with isospin, strangeness quantum numbers $(I, S)=(0,0),(1,0)$, and $(1 / 2,-1)$, corresponding respectively to $\Lambda_{c}, \Sigma_{c}$, and $\Xi_{c}$ states, the experimental information of which is gathered in Table II. We next explore the sector $(I, S)=(0,-2)$ of the $\Omega_{c}$ states, which so far shows no experimental evidence for $J^{P}=1 / 2^{-}$states. Finally, we comment on the $(I, S)=(1 / 2,1)$ sector that can only be realized with the presence of five quarks.

\section{A. $\Lambda_{c}$ resonances: $(I=0, S=0, C=1)$ sector}

In this sector, a three-star narrow $J=1 / 2^{-}$resonance exists, $\Lambda_{c}(2595)$, which has been extensively studied in various works [76-81]. We start by comparing in Fig. 2 the results obtained using our nonlocal kernel with those taking the limit $t \rightarrow 0$. We represent the imaginary part of the scattering amplitude of the elastic process $D N \rightarrow D N$ as a function of $\sqrt{s}$ for zero incoming and outgoing relative-momentum values. We can see that by adjusting the cutoff value, both models of the kernel can generate this state dynamically. However, the zero-range approximation needs a cutoff value of $\Lambda=553 \mathrm{MeV}$, whereas the finite-range interaction requires a substantially larger value of $\Lambda=903 \mathrm{MeV}$.

This is easily understood from the fact that the $D N \rightarrow D N$ diagonal matrix elements of the nonlocal potential, largely 
TABLE I. Coupled-channel meson-baryon states with charm $C=1$ and all possible combinations of isospin, strangeness $(I, S)$.

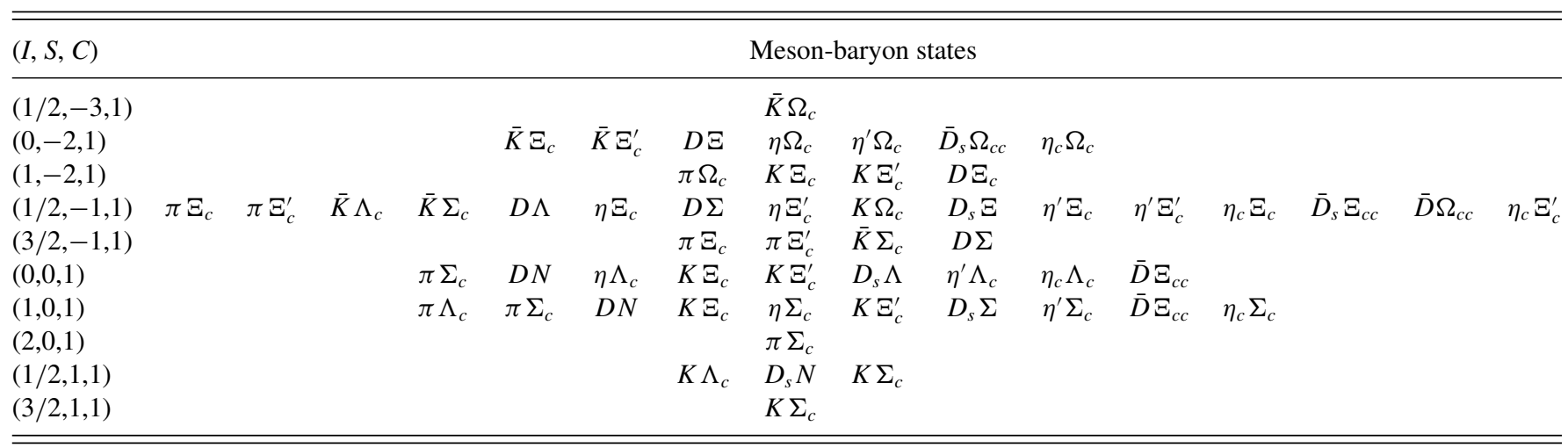

responsible for generating the resonance, are smaller in magnitude than those of the local one. The large difference between the cutoff momentum values is just a reflection of the importance of the nonlocal terms in this problem. Once the $\Lambda_{c}$ (2595) resonance is conveniently located at its experimental position by both prescriptions, substantial differences in its width remain. The local potential produces a very narrow resonance, of width $0.15 \mathrm{MeV}$, whereas the resonance generated by the finite-range potential has a width of $0.5 \mathrm{MeV}$, closer to the empirical value of $3.6+2.0-1.3 \mathrm{MeV}$. Again, this is due to the different magnitude of the nondiagonal matrix elements $D N \rightarrow \pi \Sigma_{c}$, which are larger in the finiterange approach. We note that our model does not consider the three-body decay channel $\Lambda_{c} \pi \pi$, which already represents almost one-third of the decay events [86]. We also observe that the results obtained with the low cutoff in the approximation $t=0$ for $\Lambda_{c}(2595)$ agree (mass, width, and couplings) with former studies of meson-baryon resonances of Hofmann and Lutz in Ref. [78] and Garcia-Recio et al. in Ref. [85].

Our search of resonances in this sector produces two states, which are listed in Table III, together with their widths and couplings to the various meson-baryon states. We immediately see that $\Lambda_{c}(2595)$ is basically a $D N$ state that couples very weakly to its only possible decaying channel, $\pi \Sigma_{c}$, thereby explaining its narrowness. We obtain an even narrower resonance at $2805 \mathrm{MeV}$, which is a $K \Xi_{c}$-bound system, a state also found around the same energy in Refs. [78] and [85]. Note that this resonance couples non-negligibly to $D N$, and if its location was moved upward in energy by $20-30 \mathrm{MeV}$ with a slight change of the cutoff parameter, it could explain part of the structures seen at less than $2.85 \mathrm{GeV}$ in the $D^{0} p$ invariant mass spectrum measured by the BaBar collaboration [68]. Table III also shows the results obtained with the local $t=0$ model. In spite of the fact that the second resonance appears at a greater energy, $2827 \mathrm{MeV}$, its width is narrower than in the finite-range model, confirming the trend observed for $\Lambda_{c}$ (2595).

Figure 3 shows the modulus square of the coherent sum of all transition amplitudes going to a final meson-baryon state to which the resonances can decay, namely,

$$
\sum_{M^{\prime} B^{\prime}}\left|C_{M^{\prime} B^{\prime}}^{R} T_{M^{\prime} B^{\prime} \rightarrow M B}(\sqrt{s})\right|^{2},
$$

where the values of the coefficients $C_{M^{\prime} B^{\prime}}^{R}$ would depend on the specific reaction used to excite the resonance. In this graphical example, they have all been taken to one. The amplitudes have been calculated for zero incoming and outgoing relative momentum values. To compare these to actual experiments, one should use the appropriate excitation coefficients as well as transition matrix elements going to the on-shell final momentum corresponding to the value of $\sqrt{s}$. Therefore, the results in Fig. 3 and similar ones throughout this article should be considered illustrative. The representation is split into the various energy regions where the resonances appear.

TABLE II. Masses, widths, decay modes and status of experimental charmed-baryon resonances with $J^{P}=1 / 2^{-}$or unknown, according to PDG [86].

\begin{tabular}{|c|c|c|c|c|}
\hline Resonance (MeV) & $I\left(J^{P}\right)$ & Width $(\mathrm{MeV})$ & Decay modes & Status \\
\hline$\Lambda_{c}(2595)^{+}$ & $0\left[(1 / 2)^{-}\right]$ & $3.6_{1.3}^{+2.0}$ & $\Lambda_{c} \pi \pi, \Sigma_{c} \pi$ & $* * *$ \\
\hline$\Lambda_{c}(2765)^{+}$or $\Sigma_{c}(2765)$ & $?\left(?^{?}\right)$ & $\sim 50$ & $\Lambda_{c} \pi \pi$ & $*$ \\
\hline$\Sigma_{c}(2800)$ & $1(? ?)$ & $75_{-17}^{+22}\left(\Sigma_{c}^{++}\right), 62_{-40}^{+60}\left(\Sigma_{c}^{+}\right), 61_{-18}^{+28}\left(\Sigma_{c}^{0}\right)$ & $\Lambda_{c} \pi$ & $* * *$ \\
\hline$\Xi_{c}(2790)$ & $1 / 2\left[(1 / 2)^{-}\right]$ & $<15\left(\Xi_{c}^{+}\right),<12\left(\Xi_{c}^{0}\right)$ & $\Xi_{c}^{\prime} \pi$ & $* * *$ \\
\hline$\Xi_{c}(2930)$ & $?\left(?^{?}\right)$ & $36 \pm 13$ & $\Lambda_{c} K$ & $*$ \\
\hline$\Xi_{c}(3055)$ & $?\left(?^{?}\right)$ & $17 \pm 13$ & $\Lambda_{c} \bar{K} \pi, \Sigma_{c} \bar{K}$ & $* *$ \\
\hline$\Xi_{c}(3080)$ & $1 / 2\left(?^{?}\right)$ & $5.8 \pm 1.0\left(\Xi_{c}^{+}\right), 5.6 \pm 2.2\left(\Xi_{c}^{0}\right)$ & $\Lambda_{c} \bar{K} \pi, \Sigma_{c} \bar{K}, \Sigma_{c}^{*} \bar{K}$ & $* * *$ \\
\hline$\Xi_{c}(3123)$ & $?\left(?^{?}\right)$ & $4 \pm 4$ & $\Sigma_{c}^{*} \bar{K}$ & $*$ \\
\hline
\end{tabular}




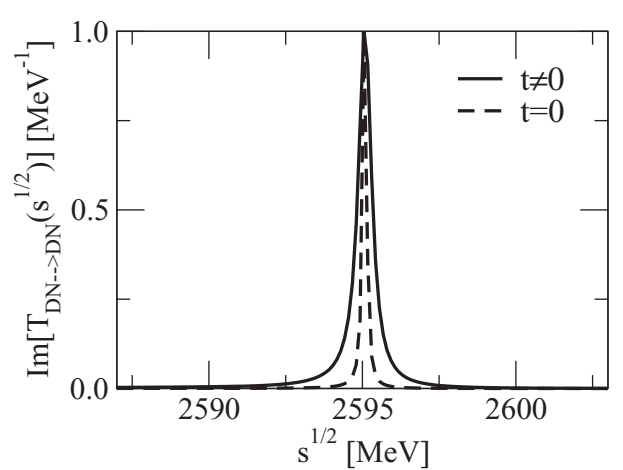

FIG. 2. Imaginary part of the scattering amplitude of the elastic process $D N \rightarrow D N$ in the $(I, S, C)=(0,0,1)$ sector as a function of $\sqrt{s}$ for the finite-range interaction (solid line) and the zero-range approximation (dashed line). The incoming and outgoing relative momenta $\vec{k}_{i}$ and $\vec{k}_{j}$ have been taken equal to 0 .

In general, a resonance couples dominantly to a given channel, and the value of the maximum of Eq. (9) is basically proportional to the modulus squared of the product of the resonance couplings to the dominant and decaying channels, $g_{M^{\prime} B^{\prime}}$ and $g_{M B}$, respectively, and inversely proportional to the resonance width $\Gamma$. Note that instead of adjusting the vertical axis of Fig. 3(b) to the maximum associated to the narrow resonance at $2805 \mathrm{MeV}$, we have scaled it down to better visualize the enhancement at $3069 \mathrm{MeV}$, right below the $K \Xi_{c}^{\prime}$ threshold. This enhancement becomes a resonance if we increase the cutoff value slightly, with properties that are similar to the state found around the same energy by the local models $[78,85]$.

\section{B. $\Sigma_{c}$ resonances: $(I=1, S=0, C=1)$ sector}

Using the same cutoff that reproduces the $\Lambda_{c}(2595)$ in the $(I, S, C)=(0,0,1)$ sector, we predict two narrow resonances at 2551 and $2804 \mathrm{MeV}$ that appear right below the thresholds of the channels to which they couple most, $\pi \Sigma_{c}$ and $D N$, respectively, as can be seen from Table IV. We also show the

TABLE III. Masses, widths, and couplings of the resonances in the $(I, S, C)=(0,0,1)$ sector, for the nonlocal $(t \neq 0)$ and local $(t=0)$ models.

\begin{tabular}{lcc|cc}
\hline \hline$\Lambda[\mathrm{MeV}]$ & \multicolumn{2}{c|}{$903(t \neq 0)$} & \multicolumn{2}{c}{$553(t=0)$} \\
$M[\mathrm{MeV}]$ & 2595 & 2805 & 2595 & 2827 \\
$\Gamma[\mathrm{MeV}]$ & 0.5 & 0.01 & 0.15 & 0.006 \\
& $\left|g_{i}\right|$ & $\left|g_{i}\right|$ & $\left|g_{i}\right|$ & $\left|g_{i}\right|$ \\
$\pi \Sigma_{c}(2591)$ & 0.44 & 0.001 & 0.21 & 0.002 \\
$D N(2806)$ & 16.34 & 0.23 & 18 & 0.03 \\
$\eta \Lambda_{c}(2832)$ & 0.58 & 1.92 & 0.22 & 1.60 \\
$K \Xi_{c}(2963)$ & 0.71 & 3.76 & 0.23 & 3.22 \\
$K \Xi_{c}^{\prime}(3070)$ & 0.32 & 0.004 & 0.01 & 0.13 \\
$D_{s} \Lambda(3085)$ & 8.16 & 0.18 & 8.54 & 0.07 \\
$\eta^{\prime} \Lambda_{c}(3243)$ & 0.96 & 0.01 & 0.54 & 0.003 \\
$\eta_{c} \Lambda_{c}(5265)$ & 2.83 & 0.02 & 1.74 & 0.007 \\
$\bar{D} \Xi_{c c}(5307)$ & 0.07 & 0.96 & 0.03 & 0.48 \\
\hline \hline
\end{tabular}
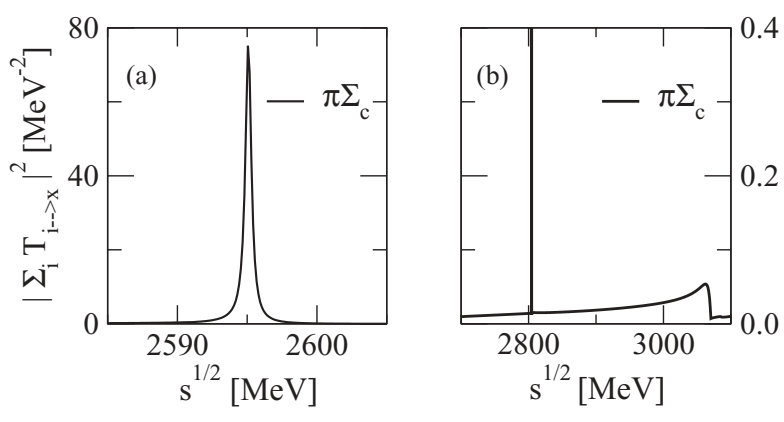

FIG. 3. Modulus square of the coherent sum of all transition amplitudes going to any of the possible final meson-baryon decaying channels, as a function of $\sqrt{s}$, for the $(I, S, C)=(0,0,1)$ sector. Our results are split into two panels, covering different energy regions and having different energy scales, to better visualize the properties of each state. The incoming and outgoing relative momenta $\vec{k}_{i}$ and $\vec{k}_{j}$ have been taken equal to zero.

results obtained with the $t=0$ model and the same cutoff value of $\Lambda=553 \mathrm{MeV}$ adjusted to the position of $\Lambda_{c}$ (2595) in the isoscalar channel. As expected, the resonance at $2804 \mathrm{MeV}$, which couples mostly to $D N$ states, barely changes its position from that of the nonlocal model. Indeed, because $\Lambda_{c}(2595)$ also couples mostly to $D N$, the cutoff adjustment of the local model using this resonance as reference has essentially left the $D N$ amplitude intact in this energy region, thereby generating similar $D N$-type bound states as in the nonlocal model in the various isospin sectors. Note, however, that the width of the resonance at $2804 \mathrm{MeV}$ is an order of magnitude smaller in the local model. The lower energy resonance, coupling mostly to $\pi \Sigma_{c}$ states, appears at somewhat larger energies in the local model, and in spite of the gain in phase space, its width is substantially reduced. This comparison confirms the trend already observed in the case of the $I=0$ sector. The resonances of the local model appear at energies similar to those of the nonlocal approach, but their widths are much smaller. Because this is a general behavior in all sectors, in the

TABLE IV. Masses, widths, and couplings of the resonances in the $(I, S, C)=(1,0,1)$ sector, for the nonlocal $(t \neq 0)$ and local $(t=0)$ models.

\begin{tabular}{lcc|cc}
\hline \hline$\Lambda[\mathrm{MeV}]$ & \multicolumn{2}{c|}{$903(t \neq 0)$} & \multicolumn{2}{c}{$553(t=0)$} \\
$M[\mathrm{MeV}]$ & 2551 & 2804 & 2585 & 2804 \\
$\Gamma[\mathrm{MeV}]$ & 0.15 & 5 & 0.005 & 0.63 \\
& $\left|g_{i}\right|$ & $\left|g_{i}\right|$ & $\left|g_{i}\right|$ & $\left|g_{i}\right|$ \\
$\pi \Lambda_{c}(2424)$ & 0.06 & 0.27 & 0.002 & 0.04 \\
$\pi \Sigma_{c}(2591)$ & 4.00 & 0.16 & 2.15 & 0.04 \\
$D N(2806)$ & 1.25 & 2.10 & 0.38 & 1.70 \\
$K \Xi_{c}(2963)$ & 0.04 & 0.20 & 0.003 & 0.06 \\
$\eta \Sigma_{c}(2999)$ & 0.79 & 0.11 & 0.44 & 0.03 \\
$K \Xi_{c}^{\prime}(3070)$ & 2.30 & 0.14 & 1.55 & 0.04 \\
$D_{s} \Sigma(3162)$ & 0.62 & 1.79 & 0.17 & 1.37 \\
$\eta^{\prime} \Sigma_{c}(3410)$ & 0.04 & 0.19 & 0.006 & 0.09 \\
$\bar{D} \Xi_{c c}(5307)$ & 0.91 & 0.15 & 0.30 & 0.02 \\
$\eta_{c} \Sigma_{c}(5432)$ & 0.13 & 0.55 & 0.02 & 0.27 \\
\hline \hline
\end{tabular}



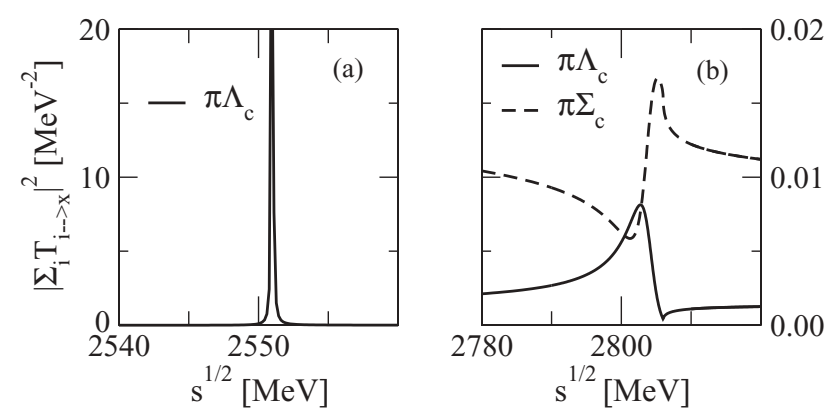

FIG. 4. Modulus square of the coherent sum of all transition amplitudes going to any of the possible final meson-baryon decaying channels, as a function of $\sqrt{s}$, for the $(I, S, C)=(1,0,1)$ sector. Our results are split into two panels, covering different energy regions and having different energy scales, to better visualize the properties of each state. The incoming and outgoing relative momenta $\vec{k}_{i}$ and $\vec{k}_{j}$ have been taken equal to zero.

remaining sections we only show the results obtained with the nonlocal approach developed in this work.

The sum of amplitudes squared is represented in Fig. 4. Because the $\Sigma_{c}(2551)$ resonance couples negligibly to its only allowed strong decaying channel, $\pi \Lambda_{c}$, it is seen in Fig. 4(a) as a narrow peak, which makes it easy to miss, given the energy resolution of the meson-baryon invariant masses built up in the experiments. The couplings to the different meson-baryon states of the $\Sigma_{c}$ resonance at $2804 \mathrm{MeV}$, visualized in Fig. 4(b), allow one to identify it with the state found in Ref. [78] at a substantially lower energy, $2680 \mathrm{MeV}$, using a subtraction method to regularize the loops, as well as with that found in Ref. [81] around $2750 \mathrm{MeV}$, using a cutoff method that preserves isospin symmetry in the regularization scheme. Our result is obviously closer to that of the latter work.

The Belle Collaboration reported recently [62] an isotriplet of excited charmed baryons, $\Sigma_{c}(2800)$, decaying into $\Lambda_{c}^{+} \pi^{-}$, $\Lambda_{c}^{+} \pi^{0}$, and $\Lambda_{c}^{+} \pi^{+}$pairs and having a width of around $60 \mathrm{MeV}$ with more than $50 \%$ error. Although this resonance has been tentatively assumed to decay to $\Lambda_{c} \pi$ pairs in the $d$-wave and its spin parity is estimated to be $J^{P}=3 / 2^{-}$, actual angular distributions have not been measured, and the fits to $\Lambda_{c} \pi$ spectra cannot rule out $s$-wave-type decays. Hence, our state at $2805 \mathrm{MeV}$ could be easily identified with the $\Sigma_{c}(2800)$ resonance, provided that three-body decay mechanisms not accounted for in our model could explain the large width observed experimentally.

\section{C. $\Xi_{c}$ resonances: $(I=1 / 2, S=-1, C=1)$ sector}

The results in this sector are presented in Table $\mathrm{V}$ and Fig. 5. We obtain two pure bound states at 2515 and $2549 \mathrm{MeV}$, respectively, which are placed less than one pion mass above the $\Xi_{c}$ member of the $J^{P}=1 / 2^{+}$ground-state antitriplet, $\left(\Lambda_{c}^{+}, \Xi_{c}^{+}, \Xi_{c}^{0}\right)$ and below the mass of the $\Xi_{c}^{\prime}$ member of the $J^{P}=1 / 2^{+}$sextet, $\left(\Sigma_{c}^{0}, \Sigma_{c}^{+}, \Sigma_{c}^{++}, \Xi_{c}^{\prime 0}, \Xi_{c}^{\prime+}, \Omega_{c}^{0}\right)$. This implies that these bound states would decay electromagnetically through the emission of $\Xi_{c} \gamma$ pairs and may have been detected at photon energies of about 50 and $80 \mathrm{MeV}$ in the experiment where $\Xi_{c}^{\prime}$ was observed [63]. Although no apparent signals
TABLE V. Masses, widths, and couplings of the resonances in the $(I, S, C)=(1 / 2,-1,1)$ sector.

\begin{tabular}{lccrrr}
\hline \hline$M[\mathrm{MeV}]$ & 2515 & 2549 & 2733 & 2840 & 2977 \\
$\Gamma[\mathrm{MeV}]$ & 0 & 0 & 34 & 0.58 & 4 \\
& $\left|g_{i}\right|$ & $\left|g_{i}\right|$ & $\left|g_{i}\right|$ & $\left|g_{i}\right|$ & $\left|g_{i}\right|$ \\
$\pi \Xi_{c}(2609)$ & 0.65 & 4.47 & 0.05 & 0.06 & 0.31 \\
$\pi \Xi_{c}^{\prime}(2715)$ & 4.84 & 0.76 & 1.77 & 0.01 & 0.22 \\
$\bar{K} \Lambda_{c}(2779)$ & 0.48 & 3.21 & 0.19 & 0.10 & 0.19 \\
$\bar{K} \Sigma_{c}(2946)$ & 6.90 & 1.01 & 7.37 & 0.93 & 0.16 \\
$D \Lambda(2985)$ & 1.03 & 0.30 & 0.96 & 1.54 & 2.95 \\
$\eta \Xi_{c}(3018)$ & 0.13 & 1.04 & 0.13 & 0.18 & 0.10 \\
$D \Sigma(3062)$ & 2.91 & 0.89 & 3.64 & 8.82 & 1.74 \\
$\eta \Xi_{c}^{\prime}(3124)$ & 4.04 & 0.59 & 3.47 & 0.46 & 0.07 \\
$K \Omega_{c}(3192)$ & 4.40 & 0.68 & 1.47 & 0.07 & 0.21 \\
$D_{s} \Xi_{(3288)}(32)$ & 1.74 & 0.15 & 0.92 & 4.71 & 2.51 \\
$\eta^{\prime} \Xi_{c}(3428)$ & 0.16 & 0.05 & 0.15 & 0.53 & 0.06 \\
$\eta^{\prime} \Xi_{c}^{\prime}(3534)$ & 0.01 & 0.03 & 0.09 & 0.11 & 0.28 \\
$\bar{D}_{s} \Xi_{c c}(5408)$ & 0.02 & 0.01 & 0.25 & 0.35 & 0.71 \\
$\bar{D} \Omega_{c c}(5429)$ & 1.10 & 0.53 & 1.18 & 0.24 & 0.03 \\
$\eta_{c} \Xi_{c}(5450)$ & 1.17 & 1.10 & 0.55 & 0.04 & 0.03 \\
$\eta_{c} \Xi_{c}^{\prime}(5556)$ & 0.04 & 0.08 & 0.26 & 0.33 & 0.82 \\
\hline \hline
\end{tabular}

were reported, we note that the limited statistical significance of the spectra measured in Ref. [63] prevents one from ruling out the existence of these bound states. Moreover, their production rate would also be somewhat inhibited by the fact that they are predominantly five-quark-component states. Note also that by lowering gradually the value of the cutoff to somewhat less than $700 \mathrm{MeV}$, the first state at $2515 \mathrm{MeV}$ eventually becomes resonant but quite narrow because of its weak coupling to the first channel, whereas the second state at $2549 \mathrm{MeV}$, which is a $\pi \boldsymbol{\Xi}_{c}$ molecule, rapidly becomes so wide that it would be difficult to distinguish it from the background.

In addition, our model gives three more resonances above the $\pi \Xi_{c}$ threshold and below $3 \mathrm{GeV}$, placed at 2733, 2840, and $2977 \mathrm{MeV}$. The local model of Ref. [78], based on on-shell amplitudes, also obtains three resonances in this energy region, located in general at somewhat lower masses and showing a different order of appearance, as can be inferred from the values of their couplings to the different meson-baryon components. More specifically, the lowest resonance in the local model appearing at $2691 \mathrm{MeV}$ and coupling strongly to $D \Sigma$ should be identified with our middle resonance at $2840 \mathrm{MeV}$. The next two resonances appear quite close in the scheme of Ref. [78], at $2793 \mathrm{MeV}$ and $2806 \mathrm{MeV}$, coupling mostly to $\bar{K} \Sigma_{c}$ and $D \Lambda$, respectively, whereas in our case they are further apart from each other, at 2733 and $2977 \mathrm{MeV}$. The crossing in the ordering of states is another consequence of the different values of the transition potential amplitudes used in both coupled-channel schemes.

Recently, several $\Xi_{c}$ states have been observed by the CLEO [64], Belle [65], and BaBar [66] Collaborations, out of which are three possible candidates to be identified with one of our states, at 2790, 2980, and $3080 \mathrm{MeV}$ (see Table II). A change of the cutoff value within a reasonable range could bring any of our two lower mass resonances to agree in position with $\Xi_{c}(2790)$, but the width would turn out to be twice as 

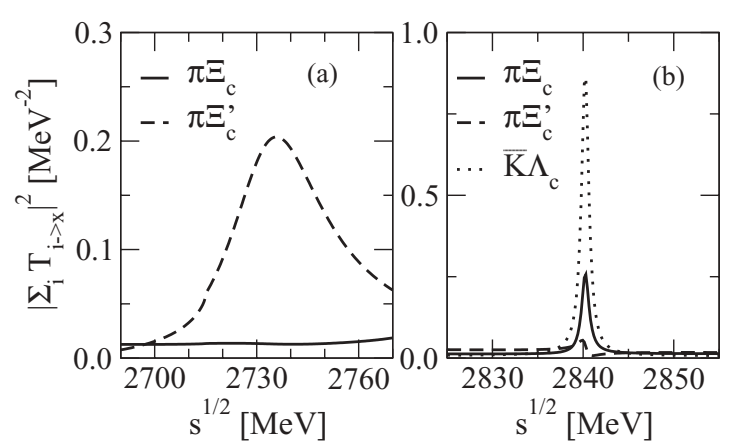

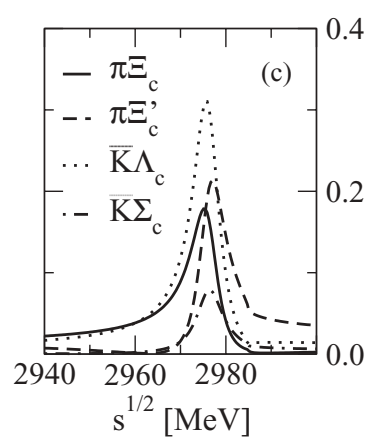

FIG. 5. Modulus square of the coherent sum of all transition amplitudes going to any of the possible final meson-baryon decaying channels, as a function of $\sqrt{s}$, for the $(I, S, C)=$ $(1 / 2,-1,1)$ sector. Our results are split into two panels, covering different energy regions and having different energy scales, to better visualize the properties of each state. The incoming and outgoing relative momenta $\vec{k}_{i}$ and $\vec{k}_{j}$ have been taken equal to zero. wide as the observed one in the case of the lower mass state. The $\Xi_{c}(2980)$ could be easily associated to either one of the two higher mass states found here. However, the authors of Ref. [66] conclude that the $\Xi_{c}(2980)^{+}$state decays in about $50 \%$ of the cases into $\Sigma_{c}^{++} K^{-}$pairs, which makes our state at $2840 \mathrm{MeV}$, showing a stronger coupling to $\bar{K} \Sigma_{c}$, the most likely candidate to be associated to $\Xi_{c}(2980)$.

\section{D. $\Omega_{c}$ resonances: $(I=0, S=-2, C=1)$ sector}

In this sector, we predict the existence of a bound state at $2959 \mathrm{MeV}$, near the lowest threshold, and two resonances placed at 2966 and $3117 \mathrm{MeV}$, as can be seen in Table VI and Fig. 6. The possible bound state could be detected through the decay into $\Omega_{c} \gamma$ states with photons of $E_{\gamma}=260 \mathrm{MeV}$ in the c.m. frame. The resonance placed at $2966 \mathrm{MeV}$ and seen in Fig. 6(a) is very narrow $(\Gamma=1.1 \mathrm{MeV})$, according to the low coupling of the resonance to the only channel in which it can decay $\left(\bar{K} \Xi_{c}\right)$ and the little available phase space. The resonance at $3117 \mathrm{MeV}$ with a width of $\Gamma=16 \mathrm{MeV}$, seen in Fig. 6(b), is a $D \Xi$ molecule that can decay into $\bar{K} \Xi_{c}$ and $\bar{K} \Xi_{c}^{\prime}$ states.

Hofmann and Lutz [78] also found three states at lower energies, 2839, 2928, and $2953 \mathrm{MeV}$, which follows the trend observed for other sectors. The pattern of couplings to the various meson-baryon states also differs a little because of the different interaction model used. The highest energy resonance in Ref. [78], coupling strongly to $\bar{K} \Xi_{c}^{\prime}$ and $\eta \Omega_{c}$, would correspond to our middle one, whereas the lowest one in Ref. [78], coupling strongly to $D \Xi$, would be the equivalent to our resonance at higher energy.

TABLE VI. Masses, widths, and couplings of the resonances in the $(I, S, C)=(0,-2,1)$ sector.

\begin{tabular}{lccr}
\hline \hline$M[\mathrm{MeV}]$ & 2959 & 2966 & 3117 \\
$\Gamma[\mathrm{MeV}]$ & 0. & 1.1 & 16 \\
& $\left|g_{i}\right|$ & $\left|g_{i}\right|$ & $\left|g_{i}\right|$ \\
$\bar{K} \Xi_{c}(2964)$ & 1.36 & 0.43 & 0.51 \\
$\bar{K} \Xi_{c}^{\prime}(3070)$ & 2.04 & 4.49 & 0.27 \\
$D \Xi(3189)$ & 2.03 & 1.68 & 5.34 \\
$\eta \Omega_{c}(3246)$ & 1.67 & 3.69 & 0.24 \\
$\eta^{\prime} \Omega_{c}(3656)$ & 0.10 & 0.07 & 0.35 \\
$D_{s} \Omega_{c c}(5528)$ & 0.17 & 1.17 & 0.19 \\
$\eta_{c} \Omega_{c}(5678)$ & 0.28 & 0.21 & 1.03 \\
\hline \hline
\end{tabular}

\section{E. Resonances of five quarks}

We have also analyzed the sectors corresponding to resonances that cannot be realized with only three quarks, and therefore their existence would signal the presence of pure five-quark states. Note that the possible pentaquark-type systems predicted by the present model would be color singlet states built up from combinations of color singlet $q \bar{q}$ with color singlet $q q q$ components. States with a different composition, such as a combination of color octet $q \bar{q}$ with color octet $q q q$ clusters, cannot be generated by our meson-baryon scattering approach.

Out of the three possible sectors, namely $(I, S, C)=$ $(2,0,1),(1 / 2,1,1)$ and $(3 / 2,1,1)$, we find only hints of a possible resonance in the case $I=1 / 2, S=1, C=1$, where we see a cusplike structure placed at the threshold of the $K \Sigma_{c}$ channel to which the state couples more strongly. This behavior is shown by the solid line in Fig. 7 and by the first column of coupling constants displayed in Table VII, which have been obtained using our nominal cutoff value of $903 \mathrm{MeV}$. According to the mechanism discussed in Ref. [44], the coupling constants should vanish if the resonance was placed right at the $K \Sigma_{c}$ threshold, which explains the small size of their values. To see whether the cusp structure would eventually become a clear resonance with a slight change of parameters, we also display in Fig. 7 and in Table VII our results with two other values of the cutoff, 1200 and $1400 \mathrm{MeV}$. One can clearly see that the cusp structure becomes a more
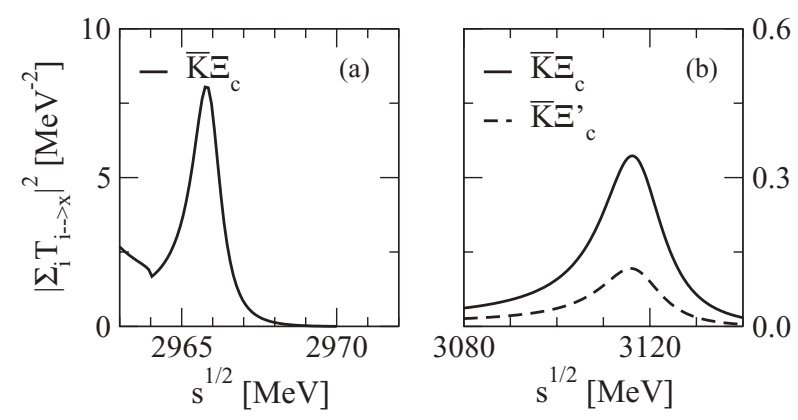

FIG. 6. Modulus square of the coherent sum of all transition amplitudes going to any of the possible final meson-baryon decaying channels, as a function of $\sqrt{s}$, for the $(I, S, C)=(0,-2,1)$ sector. Our results are split into two panels, covering different energy regions and having different energy scales, to better visualize the properties of each state. The incoming and outgoing relative momenta $\vec{k}_{i}$ and $\vec{k}_{j}$ have been taken as equal to zero. 
TABLE VII. Masses, widths, and couplings of the resonances in the $(I, S, C)=(1 / 2,1,1)$ sector for different cutoff values: 903, 1200 , and $1400 \mathrm{MeV}$.

\begin{tabular}{lcrr}
\hline \hline$\Lambda[\mathrm{MeV}]$ & 903 & 1200 & 1400 \\
$M[\mathrm{MeV}]$ & 2946 & 2941 & 2924 \\
$\Gamma[\mathrm{MeV}]$ & 0.93 & 5 & 12 \\
& $\left|g_{i}\right|$ & $\left|g_{i}\right|$ & $\left|g_{i}\right|$ \\
$K \Lambda_{c}(2779)$ & 0.002 & 0.04 & 0.10 \\
$D_{s} N(2908)$ & 0.03 & 0.84 & 1.68 \\
$K \Sigma_{c}(2946)$ & 0.07 & 1.79 & 3.59 \\
\hline \hline
\end{tabular}

bound and wider resonance as the cutoff value increases, while the coupling constants become larger.

\section{F. Dependence on model parameters}

We finalize this work by exploring the dependence of our results on the shape and size of the form factor employed, which are ingredients of the model that are not constrained by symmetry arguments.

First, by replacing the dipole-type form factor by a Gaussian form,

$$
F(|\vec{k}|)=\mathrm{e}^{-\frac{\vec{k}^{2}}{2 \Lambda_{g}^{2}}}
$$

we are able to adjust the position of $\Lambda_{c}(2595)$ with a Gaussian cutoff value of $\Lambda_{g}=543 \mathrm{MeV}$. The corresponding width is exactly the same as that found for the dipole-type form factor. When exploring the other sectors, the Gaussian form factor gives rise to the same resonances, some of them slightly displaced by at most $50 \mathrm{MeV}$ from the position found with the dipole-type form factor but having essentially the same width.

Retaining the dipolar form factor, we next explore the effects of varying the value of the cutoff $\Lambda$ between 600 and $1200 \mathrm{MeV}$, that is, up to $300 \mathrm{MeV}$ below and above the nominal value of $903 \mathrm{MeV}$ used in this work. This variation produces changes in the positions and widths of the resonances within

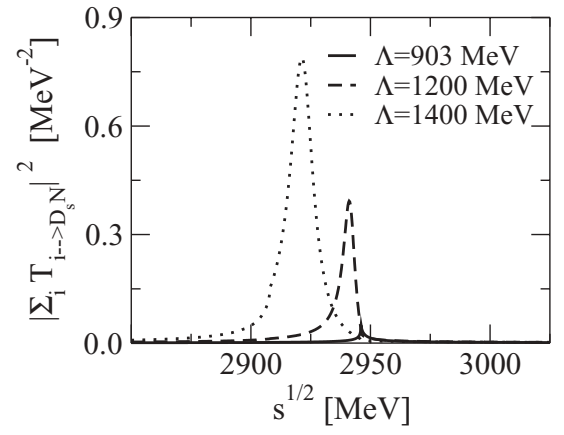

FIG. 7. Modulus square of the coherent sum of all transition amplitudes going to any of the possible final meson-baryon decaying channels, as a function of $\sqrt{s}$, for the $(I, S, C)=(1 / 2,1,1)$ sector for three different cutoff values: 903,1200 , and $1400 \mathrm{MeV}$. Our results are split into two panels, covering different energy regions and having different energy scales, to better visualize the properties of each state. The incoming and outgoing relative momenta $\vec{k}_{i}$ and $\vec{k}_{j}$ have been taken as equal to zero.

certain ranges, the general trends of which are summarized in the following points:

(i) A resonance that lies far below-by 50 to $200 \mathrm{MeV}$ the meson-baryon threshold to which it couples more strongly may change its position by an amount comparable with the variation of the cutoff value. The larger the cutoff, the more bound the resonance becomes.

(ii) The width of the resonance changes appreciably only for cutoff values that move the resonance above the threshold of a meson-baryon channel to which the resonance couples significantly.

(iii) Weakly bound resonances change their positions more moderately, at most by $10 \mathrm{MeV}$, for changes of cutoff values within $100 \mathrm{MeV}$. In this case, the width tends to decrease as the resonance becomes less bound because of the distortions induced by moving closer to the threshold, a phenomenon also known as the Flatté effect [92].

TABLE VIII. Masses, widths, and main coupled channels of states that can be identified with well-established resonances in various sectors.

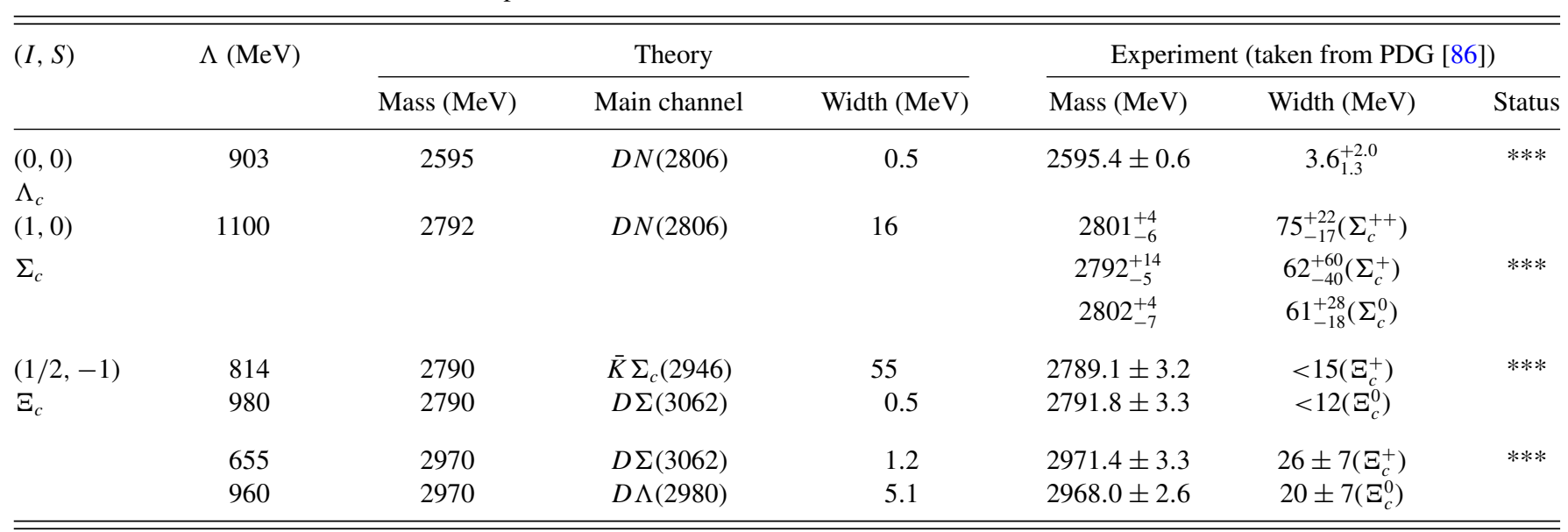


Having explored the systematics to the cutoff changes, we finally summarize in Table VIII the states that, taking an appropriate cutoff value within the range explored, could be identified with a well-established resonance of $J^{P}=1 / 2^{-}$or unknown spin parity.

\section{CONCLUSIONS}

We have studied charmed-baryon resonances obtained by a coupled-channel unitary approach using a $t$-channel vectorexchange driving force.

To the best of our knowledge, all previous models of dynamically generated baryon resonances in the charm sector rely on a local zero-range interaction, which is obtained by neglecting the four-momentum transfer $t$ in front of the mass of the exchanged vector meson squared, $m_{V}^{2}$. However, we have illustrated that the value $t / m_{V}^{2}$ is not at all negligible in the heavy sector, especially for charm-exchange amplitudes that produce a large value of the four-momentum transfer as a result of the large difference between the masses of the mesons involved in the transition.

We have analyzed in detail the effects of going beyond the $t=0$ approximation and, taking the $I=0, C=1, J=1 / 2$ sector of the well-established $J=1 / 2^{-} \Lambda_{c}(2595)$ resonance as reference, we find that the experimental data are better reproduced by the nonlocal model, which also requires a more reasonable cutoff regularization value of $903 \mathrm{MeV}$.

Compared to the local models based on on-shell amplitudes, our approach obtains basically the same amount of resonances in all sectors but appearing, in general, at somewhat larger energies because the diagonal amplitudes, largely responsible for generating the bound states, are smaller in magnitude. An essential finding of this work is that our nonlocal approach produces much wider resonances because of the larger value of the nondiagonal amplitudes when $t \neq 0$.

By varying the cutoff parameter within a reasonable range, we are able to locate some of our states at the energy position of a measured resonance in the same sector. In particular, we suggest the identification of $\Lambda_{c}(2595), \Sigma_{c}(2800), \Xi_{c}(2790)$, and $\Xi_{c}(2980)$ as dynamically generated resonances having $J^{P}=1 / 2^{-}$. In general, the widths of the states produced by our model are smaller than the experimentally observed ones, because we do not account for three-particle decay channels.

We find a possible resonance in the sector with quantum numbers $(I, S, C)=(1 / 2,1,1)$ that can only be realized by the consideration of a minimum of five quarks. The cusplike structure observed at the threshold of the $K \Sigma_{c}$ channel for a cutoff value of $903 \mathrm{MeV}$ becomes a more bound and wider clear resonance as the cutoff value increases.

This is the first exploratory study of the effects tied to the nonlocality of the meson-baryon interaction in the charm sector. We have considered meson-baryon coupled states built up from the $J^{P}=0^{-}$mesons and the ground state $J^{P}=1 / 2^{+}$ baryons. However, heavy-quark symmetry demands that the states containing heavy vector mesons are treated on equal footing because of the similarity of their masses with the heavy pseudoscalar ones. This will be addressed in a future work.

\section{ACKNOWLEDGMENTS}

We are very grateful to M. F. M. Lutz for helpful discussions and comments. We also thank E. Graugés for clarification of the details of the experiments and B. Juliá-Díaz, V. K. Magas, and A. Parreño for interesting suggestions and their help in various stages of the calculation. This work was partly supported by European Union (EU) Contract No. MRTN-CT-2006-035482 (FLAVIAnet), Ministerio de Ciencia e Innovación (MICINN, Spain) Contract No. FIS2008-01661, the Generalitat de Catalunya Contract No. 2009SGR-1289, and Fundação para a Ciência e a Tecnologia (FCT/FEDER, Portugal) under the project CERN/FP/83505/2008. We acknowledge the support of the European Community-Research Infrastructure Integrating Activity "Study of Strongly Interacting Matter" (HadronPhysics2, Grant Agreement No. 227431) under the Seventh Framework Programme of the EU.
[1] J. S. Ball and W. R. Frazer, Phys. Rev. Lett. 7, 204 (1961).

[2] H. W. Wyld, Phys. Rev. 155, 1649 (1967).

[3] R. H. Dalitz, T. C. Wong, and G. Rajasekaran, Phys. Rev. 153, 1617 (1967).

[4] R. K. Logan and H. W. Wyld, Phys. Rev. 158, 1467 (1967).

[5] G. Rajasekaran, Phys. Rev. D 5, 610 (1972).

[6] P. B. Siegel and W. Weise, Phys. Rev. C 38, 2221 (1988).

[7] N. Kaiser, P. B. Siegel, and W. Weise, Nucl. Phys. A594, 325 (1995); Phys. Lett. B362, 23 (1995); N. Kaiser, T. Waas, and W. Weise, Nucl. Phys. A612, 297 (1997).

[8] J. Nieves and E. Ruiz Arriola, Phys. Rev. D 63, 076001 (2001).

[9] C. Garcia-Recio, J. Nieves, E. R. Arriola, and M. J. V. Vacas, Phys. Rev. D 67, 076009 (2003).

[10] A. Ramos, E. Oset, and C. Bennhold, Phys. Rev. Lett. 89, 252001 (2002).

[11] E. Oset, A. Ramos, and C. Bennhold, Phys. Lett. B527, 99 (2002).

[12] D. Jido, J. A. Oller, E. Oset, A. Ramos, and U. G. Meissner, Nucl. Phys. A725, 181 (2003).
[13] E. Oset and A. Ramos, Nucl. Phys. A635, 99 (1998).

[14] U. G. Meissner and J. A. Oller, Nucl. Phys. A673, 311 (2000).

[15] J. A. Oller, E. Oset, and A. Ramos, Prog. Part. Nucl. Phys. 45, 157 (2000).

[16] J. A. Oller and U. G. Meissner, Phys. Lett. B500, 263 (2001).

[17] J. Nieves and E. R. Arriola, Phys. Rev. D 64, 116008 (2001).

[18] M. F. M. Lutz and E. E. Kolomeitsev, Nucl. Phys. A700, 193 (2002).

[19] T. Inoue, E. Oset, and M. J. Vicente Vacas, Phys. Rev. C 65, 035204 (2002).

[20] J. A. Oller, J. Prades, and M. Verbeni, Phys. Rev. Lett. 95, 172502 (2005).

[21] B. Borasoy, R. Nissler, and W. Weise, Eur. Phys. J. A 25, 79 (2005).

[22] B. Borasoy, U. G. Meissner, and R. Nissler, Phys. Rev. C 74, 055201 (2006).

[23] T. Hyodo, D. Jido, and A. Hosaka, Phys. Rev. C 78, 025203 (2008).

[24] T. Hyodo, D. Jido, and L. Roca, Phys. Rev. D 77, 056010 (2008). 
[25] S. Capstick and N. Isgur, Phys. Rev. D 34, 2809 (1986).

[26] P. Gonzalez, J. Vijande, and A. Valcarce, Phys. Rev. C 77, 065213 (2008).

[27] V. K. Magas, E. Oset, and A. Ramos, Phys. Rev. Lett. 95, 052301 (2005).

[28] D. Jido, E. Oset, and T. Sekihara, Eur. Phys. J. A 42, 257 (2009).

[29] D. W. Thomas, A. Engler, H. E. Fisk, and R. W. Kraemer, Nucl. Phys. B56, 15 (1973).

[30] S. Prakhov et al. (Crystal Ball Collaboration), Phys. Rev. C 70, 034605 (2004).

[31] O. Braun et al., Nucl. Phys. B129, 1 (1977).

[32] A. Le Yaouanc, L. Oliver, O. Pene, and J. C. Raynal, Hadron Transitions of the Quark Model (Gordon and Breach, Amsterdam, 1988).

[33] R. Koniuk and N. Isgur, Phys. Rev. D 21, 1868 (1980) [Erratum-ibid. 23, 818 (1981)].

[34] N. A. Törnqvist and P. Żenczykowski, Phys. Rev. D 29, 2139 (1984).

[35] N. A. Törnqvist and P. Żenczykowski, Z. Phys. C 30, 83 (1986).

[36] W. Blask, M. G. Huber, and B. Metsch, Z. Phys. A 326, 413 (1987).

[37] B. Silvestre-Brac and C. Gignoux, Phys. Rev. D 43, 3699 (1991).

[38] E. E. Kolomeitsev and M. F. M. Lutz, Phys. Lett. B585, 243 (2004).

[39] S. Sarkar, E. Oset, and M. J. Vicente Vacas, Nucl. Phys. A750, 294 (2005) [Erratum-ibid. A780, 90 (2006)].

[40] L. Roca, S. Sarkar, V. K. Magas, and E. Oset, Phys. Rev. C 73, 045208 (2006).

[41] M. Doring, E. Oset, and D. Strottman, Phys. Lett. B639, 59 (2006).

[42] M. F. M. Lutz, G. Wolf, and B. Friman, Nucl. Phys. A706, 431 (2002).

[43] C. García-Recio, J. Nieves, and L. L. Salcedo, Phys. Rev. D 74, 034025 (2006).

[44] H. Toki, C. Garcia-Recio, and J. Nieves, Phys. Rev. D 77, 034001 (2008).

[45] P. Gonzalez, E. Oset, and J. Vijande, Phys. Rev. C 79, 025209 (2009).

[46] S. Sarkar, B. X. Sun, E. Oset, and M. J. V. Vacas, arXiv:0902.3150 [hep-ph].

[47] E. Oset and A. Ramos, arXiv:0905.0973 [hep-ph].

[48] A. Martinez Torres, K. P. Khemchandani, and E. Oset, Phys. Rev. C 77, 042203(R) (2008).

[49] A. Martinez Torres, K. P. Khemchandani, and E. Oset, Eur. Phys. J. A 35, 295 (2008).

[50] K. P. Khemchandani, A. Martinez Torres, and E. Oset, Eur. Phys. J. A 37, 233 (2008).

[51] D. Jido and Y. Kanada-En'yo, Phys. Rev. C 78, 035203 (2008).

[52] Y. Kanada-En'yo and D. Jido, Phys. Rev. C 78, 025212 (2008).

[53] M. F. M. Lutz and E. E. Kolomeitsev, Found. Phys. 31, 1671 (2001).

[54] M. F. M. Lutz, GSI-Habil-2002-1.

[55] M. F. M. Lutz and E. E. Kolomeitsev, Nucl. Phys. A730, 392 (2004).

[56] B. Aubert et al. (BaBar Collaboration), Phys. Rev. Lett. 90, 242001 (2003).

[57] D. Besson et al. (CLEO Collaboration), Phys. Rev. D 68, 032002 (2003) [Erratum-ibid. 75, 119908 (2007)].

[58] P. Krokovny et al. (Belle Collaboration), Phys. Rev. Lett. 91, 262002 (2003).
[59] S. K. Choi et al. (Belle Collaboration), Phys. Rev. Lett. 91, 262001 (2003).

[60] D. E. Acosta et al. (CDF II Collaboration), Phys. Rev. Lett. 93, 072001 (2004).

[61] M. Artuso et al. (CLEO Collaboration), Phys. Rev. Lett. 86, 4479 (2001).

[62] R. Mizuk et al. (Belle Collaboration), Phys. Rev. Lett. 94, 122002 (2005).

[63] C. P. Jessop et al. (CLEO Collaboration), Phys. Rev. Lett. 82, 492 (1999).

[64] S. E. Csorna et al. (CLEO Collaboration), Phys. Rev. Lett. 86, 4243 (2001).

[65] R. Chistov et al. (BELLE Collaboration), Phys. Rev. Lett. 97, 162001 (2006).

[66] B. Aubert et al. (BaBar Collaboration), Phys. Rev. D 77, 012002 (2008)

[67] B. Aubert et al. (BaBar Collaboration), Phys. Rev. Lett. 97, 232001 (2006).

[68] B. Aubert et al. (BaBar Collaboration), Phys. Rev. Lett. 98, 012001 (2007).

[69] R. Mizuk et al. (Belle Collaboration), Phys. Rev. Lett. 98, 262001 (2007).

[70] E. E. Kolomeitsev and M. F. M. Lutz, Phys. Lett. B582, 39 (2004)

[71] J. Hofmann and M. F. M. Lutz, Nucl. Phys. A733, 142 (2004).

[72] F. K. Guo, P. N. Shen, H. C. Chiang, and R. G. Ping, Phys. Lett. B641, 278 (2006).

[73] F. K. Guo, P. N. Shen, and H. C. Chiang, Phys. Lett. B647, 133 (2007).

[74] D. Gamermann, E. Oset, D. Strottman, and M. J. V. Vacas, Phys. Rev. D 76, 074016 (2007).

[75] D. Gamermann and E. Oset, Eur. Phys. J. A 33, 119 (2007).

[76] M. F. M. Lutz and E. E. Kolomeitsev, Nucl. Phys. A730, 110 (2004).

[77] M. F. M. Lutz and E. E. Kolomeitsev, Nucl. Phys. A755, 29c (2005).

[78] J. Hofmann and M. F. M. Lutz, Nucl. Phys. A763, 90 (2005).

[79] J. Hofmann and M. F. M. Lutz, Nucl. Phys. A776, 17 (2006).

[80] L. Tolos, J. Schaffner-Bielich, and A. Mishra, Phys. Rev. C 70, 025203 (2004).

[81] T. Mizutani and A. Ramos, Phys. Rev. C 74, 065201 (2006).

[82] N. Isgur and M. B. Wise, Phys. Lett. B232, 113 (1989).

[83] M. Neubert, Phys. Rep. 245, 259 (1994).

[84] A. V. Manohar and M. B. Wise, Heavy Quark Physics, Cambridge Monographs on Particle Physics, Nuclear Physics, and Cosmology, Vol. 10 (Cambridge University Press, Cambridge, 2000).

[85] C. Garcia-Recio, V. K. Magas, T. Mizutani, J. Nieves, A. Ramos, L. L. Salcedo, and L. Tolos, Phys. Rev. D 79, 054004 (2009).

[86] Particle Data Group, C. Amsler et al., Phys. Lett. B667, 1 (2008).

[87] A. D. Lahiff and I. R. Afnan, Phys. Rev. C 66, 044001 (2002).

[88] R. Machleidt, K. Holinde, and C. Elster, Phys. Rep. 149, 1 (1987).

[89] A. Mueller-Groeling, K. Holinde, and J. Speth, Nucl. Phys. A513, 557 (1990); R. Buettgen, K. Holinde, A. MuellerGroeling, J. Speth, and P. Wyborny, ibid. A506, 586 (1990); M. Hoffmann, J. W. Durso, K. Holinde, B. C. Pearce, and J. Speth, ibid. A593, 341 (1995).

[90] J. Haidenbauer, G. Krein, U. G. Meissner, and A. Sibirtsev, Eur. Phys. J. A 33, 107 (2007).

[91] F. Gross, Phys. Rev. 186, 1448 (1969).

[92] S. M. Flatté, Phys. Lett. B63, 224 (1976). 